\title{
Author index to Volume 85
}

\section{Key to abbreviations:}

(BR) Book reviews; (L) Letters to the editors

Aalders JG 1627

Aas T 74

Abbou CC 1515

Abdulkarim B 2017

Abeliovich D 36

Adami H-O 678, 1317

Adams LM 1403

Adjei AA 484

Adjuvant Breast Cancer Project

Belgium 1

Agelaki S 798

Aguggini S 1106

Agus V 1535

Ahern R 953

A'Hern R 1383

Aherne GW 446

Ahlner-Elmqvist M 1478

Ahmad SA 584

Ahmed A 1667

Aillet G 1831

Aine R 552

Akahira J 1731

Al-Mulla F 692, 1032

Alakhov VY 1987

Ali MS 661

Allen-Mersh TG 1640

Allevi G 1106

Allinen M 209

Alman BA 98

Almonte M 966

Alonso ME 204

Alonso MM 1400

Alquati P 1106

Altman DG 944

Alvarez M 966

Amadori D 845

Amiel J 1412

Amino N 102

Ammatuna M 795

Amos CI 1037

Andersen SN 692

Anderson CD 157

Anderson H 137 (L), 1085

Anderson J 493, 831

Anderson RA 1023

Andersson S 1153

Andi A 863

Ando M 1634

Ando Y 1634

Andreyev HJN 692

Andronicos NM 909

Anissimov YG 157

Anker GB 147

Antalis TM 608

Aoki I 313
Arcangeli A 333

Ardizzoni A 1452

Arends JW 692

Arjona D 204

Armand JP 649

Armstrong BK 668

Arndt V 972

Arnold JA 297

Arnold JM 1351

Arrieta O 1396

Ascoli V 379

Asghar MS 875

Asham E 1759

Asosingh K 1387

ATAC Trialists Group 317

Ataman ÖU 1113

Atar E 504

Athanasou NA 78

Atherton PJ 152

Atzpodien J 1130

Aw SE 1978

Azuma T 692

Azzarelli A 405, 1535

Babin-Boilettot A 1646

Baccarelli A 1304

Bachelot T 816

Baek MJ 1147

Baglietto L 1838

Bagnardi V 1700

Baguley BC 687, 1219

Balaji V 705

Baldini E 1452

Bálint É 1813

Balkwill F 891

Ball HM-L 1102

Ballardini B 1838

Balsari A 1964

Balster DA 266

Bamberg M 1233

Bamberger A-M 546

Bar-Meir S 1368

Barajon I 1964

Bardelmeijer HA 1472

Barker DJP 1680

Barr MP 273

Barthelmes HU 1585

Bartlett JMS 1894

Basset-Séguin N 1883

Batlle A 279, 1794

Batrakova EV 1987

Bauer M 1540

Baxevanis CN 1527

Bayoumy S 1037

Bazan V 692
Beauduin M 1

Bebawy M 1998

Begum N-M 122

Beijnen JH 1472, 1627

Belinky A 504

Belka C 1233

Bell A 1551

Bell JA 1958

Bell S 692

Belli S 379

Bello MJ 204

Benamouzig R 692

Benedetti M 379

Bener A 1675

Benhattar J 692, 1713

Bennewith KL 1577

Benraad ThJ 85

Bensmaïne MA 509

Benson C 953

Bentzen SM 1113

Beranek M 692

Berchier MC 1444

Berdel WE 1168

Berger F 721

Berghmans T 1444

Bergkvist L 346, 869

Bergman-Jungeström M 859

Bergström A 984

Berkel HJ 991

Bernardo G 141

Berns EMJJ 538, 1359

Berrino F 785

Berruti A 1106

Bersiga A 1106

Bertani H 1607 (L)

Berthelot J-M 1280

Berthier-Vergnes O 1944

Bertoli G 1106

Bertrand S 518

Besse F 1515

Betri E 1106

Bianchi-Scarra G 836

Biddulph JP 350

Biesma B 1456

Biganzoli E 795

Bigelow CE 727

Bilim V 557, 1119

Birch JM 293

Biroccio A 1914

Biron P 816

Bjellerup P 2004

Björk P 129

Blackhall FH 1094

Bladström A 674

Blakey DC 764
Blamey RW 1958

Blangiardo M 1700

Blanks RG 1289

Blay J-Y 816

Bleiberg H 509

Blomley M 1085

Blouin M-J 428

Boddy AV 23

Boddy SC 1023

Bodis S 2010

Boege F 1585

Boffetta P 678, 1317

Bojunga J 1546

Bolsi G 1106

Boman G 1317

Bonadonna G 490

Bonanni B 1838

Bondy ML 1037

Bonfirraro G 333

Boni L 1452

Boonsong A 1492

Boracchi P 795

Borg A 1201

Borg C 816

Borri P 333

Bos AME 1627

Bosetti C 341

Bostick PJ 1340

Bottini A 1106

Botwood N 139 (L)

Botzlar A 1655

Boucaut KJ 608

Bouchardy C 1251

Bouchier-Hayes DJ 273

Boudioni M 641

Boulos PB 1759

Boulton M 641

Bounacer A 1831

Bourgain C 1592

Bourhis J 2017

Boyd A 741

Boyle JM 293

Braakhuis BJM 630

Braham D 1486

Brakenhoff RH 630

Brandl M 1655

Breivik J 692

Brekken C 1968

Bremer M 850

Brenner B 504

Brenner H 367, 972

Bret-Dibat C 1467

Bretherton-Watt D 171

Brett L 1937

Brewster DH 41, 637 
Bridle H 953

Brienza S 509

Britten R 902

Brizzi MP 1106

Brodin T 129

Brodowicz T 1764

Brooks LA 1551

Brooks SA 1014

Broome JD 930

Brorson SH 1418 (L)

Brosius M 1372

Brown G 453

Brown J 1289

Brown JE 1137

Brown P 1865

Brown RF 1273

Brugnatelli S 141

Brunelli A 1106

Brünner N 85

Brusic V 1738

Bruzzi P 1106

Bryant PE 1157

Bubb VJ 692

Bucana CD 584

Buccoliero AM 333

Buchmann J 791

Buckels J 1865

Budach W 1233

Budinsky A 1764

Budinsky AC 1850

Budman DR 1403

Buhr H-J 1540

Buley I 1014

Bundred NJ 1522

Bunyan DJ 1486

Buring JE 962

Burke D 1640

Burn J 166

Burnett RD 453

Burnstock G 1759

Burrell HC 225

Butler AR 1794

Butow PN 1273

Buzard GS 247

Byrne P 1206

Caceres E 966

Cafferata MA 1452

Calista D 1304

Calistri D 845

Cals L 649

Camlett I 1124

Canamero M 261

Cancer Research

Campaign PK/PD

Technologies Advisory

Committee 1085

Capocaccia R 785

Cappellen D 1515

Carayon P 1771

Carcenac M 1787

Carlsson P 497

Carmichael J 137 (L), 1865

Carnochan P 1640

Carrié C 1646

Carroll-Pankhurst C 1295

Carter N 171
Carton E 1499, 1781

Casadei S 845

Casas A 279, 1794

Caselmann WH 697

Cassidy J 1492

Cassiman JJ 98

Castillo M 966

Catchpoole DR 1564

Cattan N 1412

Certa U 107

Chaggar R 422

Chamberlain M 1175

Chandraratna RAS 453

Chang A 393

Charlton RG 576

Chau I 1258

Chauvin F 816

Chauzy C 1412

Chavaudra N 2017

Chazal M 439

Cheadle JP 1226

Chemotherapy

Standardisation Group of

the United Kingdom

Children's Cancer Study

Group 23

Chen C-J 658

Chen J 1047

Chen Y-L 1185

Chenevix-Trench G 687 , 1351

Cheng KK 1667

Cherubini A 333

Chetia CK 661

Cheung N-KV 182

Chevreau C 1467

Chiba T 1006

Chieffi O 333

Chilvers CED 1667

Chio C-C 1185

Cho M 563

Choma D 14

Chopin DK 1515

Chott A 1462

Choudry GA 1137

Chow W-H 1317

Christensen M 568

Christodoulakis M 798

Chughtai S 531

Cimerman N 1193

Cippitelli M 1914

Clark AB 568

Clark TG 944

Clarke PA 692

Clausen OPF 692

Clifford SC 1226

Cocker HA 1746

Colantoni A 1607 (L)

Coleman D 803

Coleman DA 1289

Coleman MG 1486

Collins S 787

Colston KW 171

Comba P 379

Connors T 1421

Connors TA 1070

Conte PF 1452
Cook AM 1624

Cook NR 962, 984

Cook-Mozaffari P 1667

Coppes RP 1055

Coradini D 795

Cornelisse CJ 1347

Corner J 1853

Corrao G 1700

Correale P 1722

Cortesi L 845

Costa J 692

Cotter TG 115

Cottu PH 1240

Coulon V 1467

Courbon F 1467

Cox G 863

Crepin M 917

Crevenna R 1850

Crichton DN 1878

Croke DT 692, 1812

Crolla J 705

Crook T 1551

Cruickshank ME 242

Cruickshank NR 692

Cull A 166, 1842

Cummings M 1351

Cunningham D 692, 1258

Curnow RT 152

Curran S 1492

Curtin JF 115

Curtin NJ 764

Cushing GL Jr 683

Cuvier C 1240

Cvitkovic E 509

Cyjon A 504

Czarnocka B 875

Dabizzi S 333

Daher A 1515

Dai Q 372

Dalgleish AG 473

Dallegri F 463

Daly JS 1499

Dame MK 1600

Dampier K 618

Damstrup L 1211

Danesi R 1425

Daniel F 1865

Danova M 141

Dapino P 463

Darmoul D 772

Daurès J-P 14

Davies EJ 1094

Davies MM 1640

Day NE 1667

De Angelis P 692

de Bock GH 1347

de Boer AC 1099

de Bruin R 1206

de Campos JM 204

De Giorgio M 1606 (L)

de Goeij AFPM 692

de Gramont A 509

De Greef C 1387

de Greve J 1

De Grève J 1592

de Jonge MJA 1124 de La Salmonière P 1883

de Lange Davies C 1968

De Maria N 1607 (L)

de Roquancourt A 1240

de Schipper FA 398

de Vries EGE 1627

de Wasch G 1

de Wit R 1099

de Witte JH 85

de Wolf C 1251

Deakin D 29

DeAngelis P 1900

Dearnaley D 1853

Debled M 1467

Decensi A 1838

Decker HJ 697

Degardin M 649

Del Bufalo D 1914

Del Tacca M 1425

Del Vecchio MT 1722

Delabie J 1900

Delaunay M 1467

Delia D 836

Della Torre G 836

Dellian M 1655

Demicheli R 490

Den Hollander JC 1557

Denic S 1675

Denys H 98

DeRenzo EG 1811 (BR)

Deutsch E 2017

Devaud H 772

Devilee P 1347

Devitt G 1372

Dewar JA 637

Dexter DW 735

Dhom G 972

Di Benedetto M 917

Di Venosa G 279

Diamandis EP 190, 220, 393

Diederichs S 1168

Dienes HP 697

Dillon D 692

Ding L 431

Ding S 1175

Dische S 1113

Dix BR 692

Dogliotti L 1106

Doherty VR 41

Dohlsten M 129

Doki Y 412

Dong C 997

Donghi R 836

Doré J-F 518

Dörk T 850

Dorr FA 1753

Dorssers LCJ 538

Dorvillius M 1787

Double JA 1137

Doubrovsky A 157

Douglas F 166

Douglas ML 608

Doyon F 1664

Drabkin HA 64

Dragan C 1546

Drazan KE 584

Drouant GJ 266 
du Vivier A 803

Duarte-Rojo A 1396

Dubost J-J 518

Dubreuil A 439

Ducreux M 509

Duda DG 431

Dumontet C 902

Duncan I 1175

Duncan P 1894

Duncan R 1070

Dunlop MG 1937

Dunn SM 1273

Dunne B 1551

Durand RE 1577

Durham J 453

Durocher F 36

Durrant S 741

Dworkin MJ 1640

Dzik-Jurasz ASK 1624

Earl H 29

East SJ 764

Easton DF 36

Ebihara Y 1706

Eccles DM 1486

Echner H 1527

Edwards DR 55

Edwards J 1894

Edwards JG 863

Edwards S 36

Edwards SM 1383

Eeles R 1383

Eeles RA 36

Eguchi K 9

Ehya H 1952

Eisen T 953

Eisenbrand G 1585

Eissa S 1037

El-Abbadi M 285

El-Badawy SA 1037

Elder JT 1600

Ellis IO 225, 1958

Ellis LM 584

Ellison D 705

Elmquist WF 1987

Elsaleh H 827

Elsby B 1332

ELYPSE Study Group 816

Encío I 1400

Endlicher E 1572

Endoh Y 199

Engels EA 1295, 1298

Engemann H 1801

Engesæter BØ 1968

Enomoto N 228

Enomoto T 247

Epstein AL 463

Eriksson JG 1680

Ernst E 781 (L)

Espenan GD 266

Espie M 1240

Etienne MC 439

European Lung Cancer Working

Party 1444

Eusebi V 422

Evans A 1289, 1551
Evans AJ 225

Evans D 1551

Evans JD 1865

Evans WK 1280

Evoy D 1781

Extra JM 1240

Fabbri A 1535

Fabbro D 2010

Faber C 692

Faithfull S 1853

Falette N 902

Fan Z 303

Fang M 303

Farinati F 1606 (L)

Farnsworth ML 1564

Farrell PJ 1551

Farrugia D 137 (L)

Fava S 141

Fayers P 1478

Fears T 1304

Ferbeye L 46

Ferguson J 139 (L)

Fernandez Garrote L 46

Fiebig H-H 1585

Fiebiger W 1462

Fiehn W 1193

Fieret JH 1359

Figer A 523, 1368

Finkelstein SD 692

Finn Bladder Group 552

Finnegan NM 115

Fiorentini S 1606 (L)

Fioretta G 1251

Fischel JL 439

Flanagan W 1280

Flannigan GM 1137

Flavell KJ 350

Flex D 523, 1368

Florin MC 1444

Florl AR 1887

Flower MA 1640

Focan C 1

Foekens JA 85, 538, 1359

Foley DA 273

FONICAP 1452

Font A 692

Forman D 1322

Formanek M 1462

Forsberg G 129

Forsén T 1680

Forsyth PA 55

Foster TH 727

Foulkes W 1383

Fowler CJ 823

Fox JC 692

Franceschi S 46, 341

Francini G 1722

Francis-Thickpenny KM 687

Franco AV 1564

François E 509

Frascona V 2017

Fredericks S 1759

French ME 1504

Fried S 1611
Friedman E 523, 1368

Friery OP 625

Frigerio S 836

Frisch M 1298

Frost T 803

Frühwald MC 1801

Fryer AA 1504

Fujikawa Y 78

Fujimori T 122, 692

Fujimoto J 313

Fujita M 247

Fujiwara Y 412

Fukuda H 279, 1794

Fulford L 422

Funke PJ 1130

Furuse K 939

Gabra H 944

Galanis E 1432

Galdos O 966

Galisson F 1883

Gallagher R 625

Gallus S 341

Galmarini CM 902

Galteland E 1900

Gamble P 1289

Gamelin E 509

Ganapathi R 747

Gao WM 235

Gao Y-T 372, 1695

Garambois V 1787

Gardaneh M 1564

Garvican L 1289

Gasali PG 1535

Gasco M 1551

Gatta G 785

Gatter KC 261, 881

Gaucherand M 1944

Gaudernack G 692

Gaulier A 1831

Gavish M 1771

Gazdar AF 1510

Gebhardt BM 266

Gedouin D 649

Geisler J 147

Geisler S 74

Geissler F 697

Gelderblom H 1124

Gemmill RM 64

Generali D 1106

Gentet JC 1646

Genus T 531

Georgoulias V 798

Gerald WL 182

German Cooperative Renal

Carcinoma Chemo-

Immuno-therapy Trials

Group 1130

Gescher A 618

Geva R 523, 1368

Ghanem MA 1557

Ghesquière H 816

Giaccone G 1456

Gianni S 1606 (L)

Giardina G 141

Giaretti W 692
Giatromanolaki A 881

Gibbs A 787

Gil M 1400

Gil-Diez-de-Medina S 1515

Gilbertson R 705

Gilmore AP 1522

Giner V 1444

Gioanni J 1412

Giordano M 141

Giorgi G 1722

Giovannucci E 346

Giuliano AE 1340

Given-Wilson R 171

Glaesener R 692

Glanzmann C 2010

Glaser B 36

Glaussel F 1787

Gleig A 1157

Glennie MJ 1619

Glimelius B 1265

Glover C 1640

Gnanapragasam VJ 1928

Gnant M 1850

Gneiting T 1655

Goedert JJ 337, 1295, 1298

Goemann C 546

Goetz AE 1655

Goh H-S 692

Goh L-b 1175

Göhring B 924

Going JJ 1894

Goldbohm RA 977

Goldgar D 1383

Goldstein AM 527

Gong Y 1706

Gonzalez-Gomez P 204

Goodwin LO 930

Gordon T 493, 831

Gore ME 953

Gough AC 1486

Gould A 1842

Gould S 831

Graham-Brown R 803

Gramatzki M 1130

Grasso D 141

Gratio V 772

Grazia Daidone M 795

Greaves MJ 293

Grebenchtchikov N 85

Greenberg ER 683

Greif F 504

Griebel J 1655

Grieu F 827

Griffin M 1781

Grigor KM 1894

Grimm J 791

Gritzapis AD 1527

Groft LL 55

Gronchi A 1535

Groves FD 337

Gualde N 1467

Gubala E 875

Guenther U 1540

Guerrero I 966

Guerrieri-Gonzaga A 1838

Guerts-Moespot A 85 
Guevara P 1396

Gustafsson B 1871

Gusterson B 1551

Haber M 1564

Haboubi NY 787

Haddad G 1219

Hadjiloucas I 1522

Hainaut P 721

Haites NE 242, 1492

Hajian-Tilaki KO 1671

Håkansson A 1871

Håkansson L 1871

Hakulinen T 357, 367

Halgunset J 1968

Hall CN 692

Hall DMS 1014

Halter SA 692

Hamilton SR 1037

Hamilton Stewart PA 1137

Hammond LA 453

Hamoudi RA 1383

Hansen LRT 568

Hansson E 552

Hara N 557, 1119

Harada M 9

Hardoff R 504

Harita S 9

Harland SJ 823

Harmey JH 273

Harris A 78

Harris AL 881

Hasegawa J 1510

Hasegawa K 939

Hasegawa Y 102, 1634

Hashida H 1922

Hashimoto I 93

Haskill J 668

Hassan M 2004

Haug I 1968

Hausmann F 1572

Hawkins N 692

Hawkins R 140 (L)

Hédelin G 808

Heemann U 1047

Hegyi I 2010

Heinzel S 1527

Hejna M 1764

Heldin P 600

Heliövaara M 357

Helle SI 74, 147

Hemmi H 1064

Hemminki K 997

Hennis B 1124

Henzen-Logmans SC 1359

Herbst H 1540

Hernan R 705

Herrero R 46

Hess C 2010

Heuvel JJTM 85

Heuze A 764

Hicklin DJ 584

Hickson ID 261

Hida Y 1922

Higgins C 608

Hilakivi-Clarke L 1680
Hill C 1664

Hill L 493

Hill M 1258

Hino S 122

Hirakawa K 612

Hiraki S 9

Hirano T 1706

Hirao Y 563

Hiraoka K 1922

Hirata K 752

Hirst DG 625

Hjelmqvist B 1871

Ho C-K 658

Ho JWC 692

Hobbs SM 1746

Hocker JE 1564

Hoffman K 1265

Hoffmann I 692

Hofstra LS 1627

Hogg D 527

Hollis R 23

Holly JMP 74, 147

Holm L-E 362

Holmberg E 362

Holmberg L 346, 869

Holte H 1900

Hongo A 93

Hoon DSB 1340

Höpfner M 1771

Hopwood P 137 (L), 166

Hoque MO 122

Horton C 325

Hoshai H 1064

Houlihan PS 1037

House A 1842

Howe K 1824

Howells LM 618

Howie AJ 152

Hsieh C-C 984

Hsu H-K 658

Hsu T 1219

Hubert A 36

Huddart R 213, 1853

Huddart RA 1624

Hudes GR 735

Hudson EA 618

Hughes CM 625

Huisman C 1456

Hunt D 705

Hunt NCA 78

Hunter JAA 803

Huot TJG 836

Hupp TR 1102, 1878

Huusko P 209

Huynh KT 1340

Hynes NE 1787

Iacopetta B 827

Iacopetta BJ 692

Ikeda N 1706

Illiger HJ 1130

Imamura M 1006

Imrie CW 1865

Inai M 1006

Ingvar C 674

Inoue H 713
Inoue S 1731

Irmin L 523, 1368

Isbert C 1540

Ishihara S 741

Ishikawa T 612

Ishikawa Y 741

Isla A 204

Islam-Ali B 758

Isola JJ 1201

Issa J-P 930

Italian Lung Cancer Task

Force (FONICAP) 1452

Itoh T 1922

Itonaga I 78

Izumi N 228

Jabbour HN 1023

Jackman AL 446

Jäger U 1462

Jaiswal AS 898

James ND 152

Jan P 1664

Jandik P 692

Janin A 1883

Janota-Bzowski M 875

Jansen A 1771

Jansen SE 1472

Järvinen R 357

Jarzab B 875

Jaunin P 1713

Jayson GC 1094

Jefferies S 1383

Jin F 372

Jin Z 69

Jodrell DI 1937

Johannsson O 1201

Johansson H 1838

Johns LE 1289

Johnson CD 1865

Johnson PWM 1619

Johnson R 649

Johnsson A 1206

Johnston PG 1937

Johnston RN 55

Jones C 422

Jones EL 453

Jones J 152

Jones JL 863

Jones PW 1504

Jones-Wallace D 705

Jordhøy MS 1478

Joseph D 827

Jozefonvicz J 917

Juji T 741

Jullian E 692

Jung K 220, 393

Jung YD 584

Kaasa S 1478

Kaasinen E 552

Kabanov AV 1987

Kadouri L 36

Kajita T 255

Kakolyris S 798

Kalbakis K 798

Kamei H 9
Kameshima H 752

Kampinga HH 1055

Kandioler D 1764

Kang H 1147

Kano M 1006

Kao E-L 658

Karagas MR 683

Karjalainen-Lindsberg M-L 383

Karlsson P 362

Karstens JH 850

Kasahara T 557, 1119

Kasamon YL 1738

Kashiwaba M 69

Kato H 1706

Kato Y 1706

Katoh H 1922

Katoh K 1922

Katsaros D 190

Kawabata W 1731

Kawada Y 563

Kawahara M 939

Kawamata H 122

Kawano K 247

Kawasaki H 1510

Kawasaki T 557

Kayhty H 337

Kayitalire L 649

Kayser K 1193

Keeling PWN 1499

Kelland LR 1746

Kelleher D 1499

Kelsey A 831

Kemp EH 875

Kempski H 493

Keohavong P 235

Kernohan N 1878

Kernohan NM 1102

Khan S 758

Khatun S 313

Kherfellah D 1077

Kierstead LS 1738

Kikuchi A 741

Kim H 1147

Kim S-C 563

Kim SE 1147

Kimura F 1887

Kimura K 255

Kimura W 199

Kirchner H 1130

Kirkwood JM 1738

Kirsten ras In-Colorectal-Cancer Collaborative Group 692

Kishi K 412

Kishimoto Y 1510

Kiura K 9

Klaassen I 630

Klastersky J 1444

Klein G 1952

Klein Kranenbarg E 780 (L)

Kleinerman ES 747

Klevenvall L 2004

Klijn JGM 85, 538, 1359

Klinzig F 808

Klug S 966

Klümpen S 1168

Knäuper V 55 
Knekt P 357

Knowles MA 1887

Knüchel R 1572

Knuechel R 727

Kobayashi D 752

Kodama J 93

Kodama T 43

Koduru P 930

Koelbl H 791

Koestler W 1764

Koezuka Y 741

Kögel D 1801

Kogner P 2004

Koi M 1064

Konaka C 1706

Kondo S 1922

Konings AWT 1055

Konishi N 563

Koopman FJ 1472

Kopchick JJ 428

Kopitar G 1193

Kopp-Schneider A 1372

Koren G 1611

Kortmann RD 1233

Kos J 1193

Kote-Jarai Z 36

Koukourakis MI 881

Koumakis G 1444

Kourbali Y 917

Kouroussis Ch 798

Kozikowski AP 1771

Kraemer M 917

Krainer M 1764

Kreis W 1403

Kricker A 668

Krieg R 1572

Krtolica K 692

Krul MRL 1137

Krysander L 1871

Kudo T 93

Kudoh S 939

Kügler K 1168

Kulkarni S 325

Kuma K 102

Kunkel TA 568

Kunstfeld R 1764

Kurita Y 1326

Kurtaran A 1462

Kusak ME 204

Kushi LH 372

Kusterer K 1546

Kyriakopoulou L 190

La Vecchia C 341, 1700

Laburthe M 772

Lachlan G 1322

Lafitte JJ 1444

Lafleur MA 55

Lagergren J 1317

Lagrange J-L 1412

Laguerre B 649

Lakhani SR 422

Landi G 1304

Landi MT 1304

Lando PA 129

Lang SH 590
Langdon SP 1753

Lange D 875

Langlois R 1787

Lantieri F 836

Lantzsch T 791

Larroque Ch 1787

Laslo D 1611

Latz J 649

Laube F 924

Launonen V 209

Laurence V 1240

Laurent-Puig P 692

Le Deley MC 1646

Le Frère-Belda MA 1515

Le Pelletier F 1883

Leach MO 1085

Lebitasy MP 808

LeCesne A 816

Lecomte J 1444

Lee C-J 658

Lee I-M 962

Lee J-M 658

Lee JQ 692

Lee JS 1147

Lee L 1289

Lee MC 285

Lee Y-C 658

Lees N 692

Lefebvre JL 649

Leirvaag B 147

Leitch A 1937

Lence Anta J 46

Lerebours F 1240

Lessells AM 1937

Leung HY 576, 1928

Levi F 341

Lévi F 509

Levin B 1037

Levine PH 337

Levrat C 902

Lewis R 137 (L)

Leydon G 641

Leyvraz S 1713

Li C 98

Li S 1987

Li Y 600

Liestø1 K 1418 (L)

Lin S-J 1185

Lin VC-L 1978

Lindblad P 984

Lishner M 1611

Lister TA 29

Liston J 1289

Liu B 303

Liu L 527

Liu S 1335, 1504

Liu W 584

Liu XM 1403

Lo Vullo S 1535

Lobelle JP 1

Lock RB 1564

Locker GJ 1850

Logan DM 1280

Logan RFA 1667

Loge JH 1478

Loizidou M 1759
Lomas J 204

Lombard-Bohas C 721

Longley DB 1937

Loni L 1425

Löning T 546

Lønning PE 74, 147

Look MP 85, 1359

Loos WJ 1124

Lorite MJ 297

Los G 1206

Losi L 692

Louhelainen J 1887

Love DR 687

Love SB 29

Lövig T 692

Lozzi L 1722

Lu C-M 984

Lu Y 303 (two)

Lu Y-J 213

Lubin R 1883

Ludwig C 1764

Luketich JD 235

Lukish JR 1037

Lund Nilsen TI 959

Lund P 697

Lundell M 362

Lunec J 705

Lung Cancer Working Group 939

Luo L-Y 220

Luoto R 1680

Luscombe CJ 1504

Maas S 1887

Maaser K 1771

McCauley RA 735

McConville CM 531

McCready VR 1640

McDowell H 831

McDowell HE 1878

McFadyen MCE 242

McFarlane G 1322

McIntosh RS 875

McKay JA 1492

McKeage MJ 1219

McKeown SR 625

McKinney PA 1667

McLaren A 1175

McLeod HL 242, 1492

McManus A 831

McManus R 1499

McPherson K 166, 641

McPhillips F 1753

Mady HH 235

Mæhlen J 1418 (L)

Maggiorella L 2017

Magois C 1831

Mahanta J 661

Maher ER 1226

Maitland NJ 590

Majois F 1

Mak I 953

Makke J 1240

Malik S 692

Malingré MM 1472

Mamalaki A 1527

Mammen A 1952
Mancini M 463

Mann JR 350, 531

Manno M 1607 (L)

Mansi JL 171

Manson MM 618

Mantel MA 1124

Mäntyniemi S 209

Mao Y 1335

Mapp T 831

Marangoni E 2017

Marchetti S 439

Mari B 1412

Mariani L 1535

Marie J-C 772

Marko D 1585

Marolleau JP 1240

Marsden RA 803

Marsh S 1492

Marshall GM 1564

Marsili S 1722

Martel-Planche G 721

Martínez-Merino V 1400

Marty M 509, 1240

Marumo F 228

Mashino K 713

Massey A 1258

Matsui K 939

Matsuno S 431

Mattsson A 362

Maughan TS 1422

Mavroudis D 798

Mayeur D 1240

Maynard JH 1226

Mazeau C 1412

Mech P 1801

Mechler C 1831

Mechniaud F 1646

Mehta J 325

Meijer C 966

Meijer CJLM 398

Meijer-van Gelder M 538

Meijers-Heijboer H 538

Melhem MF 235

Melia J 641, 656, 803

Meling GI 692

Melvin WT 242

Ménard S 1964

Mende T 791

Merrett S 422

Merry CLR 1094

Messmann H 1572

Methner C 546

Metzger R 1168

Metzner B 1130

Meyer L 1853

Mezzelani A 405, 1535

Michalski A 493

Michel J 1444

Micheli L 1722

Michon J 1646

Miclea JM 1240

Migliaccio M 1400

Milano G 439

Milde-Langosch K 546

Miller DW 1987

Miller ID 242 
Miller K 1759

Milliken D 891

Mills GB 303

Milne SA 1023

Minami H 1634

Miodini P 795

Misawa H 1326

Mitra S 727

Miura N 1510

Miyamoto M 1922

Miyasaka Y 228

Miyata H 412

Miyauchi A 102

Moch C 1883

Mocroft A 1824

Mokhtar N 1037

Möller M 1168

Möller TR 674

Mommen P 1444

Monden M 412

Monia BP 1753

Monks NR 764

Montanari G 141

Montaudon D 1077

Moon SE 1600

Mora J 182

Moreau L 808

Morelli D 1964

Morgan B 115

Mori M 713

Moriya T 1731

Morris A 297

Morris MB 1998

Morrissey C 1226

Mortimer EA Jr 1295

Morton D 692

Moss S 656, 803

Moss SM 1289

Mossman J 641

Motoyama T 69, 199

Mott LA 683

Moynihan C 641

Moysan A 1883

MPT Collaborators 1383

Mucci LA 678

Muehlbacher F 1850

Mulder NH 1627

Mullen P 1753

Muller O 692

Müller-Tidow C 1168

Mulligan E 1781

Multani AS 898

Muñoz N 46, 966

Muramatsu M 1731

Murata Y 247

Murawaki Y 1510

Murdoch J 1824

Murphy KM 1564

Murray GI 242, 1492

Murray MM 625

Murray PG 350

Muzik H 55

Mycroft J 23

NACCP Group 1437

Nagashima H 713
Nagayama K 228

Nakagawa H 412

Nakakubo Y 1922

Nakamoto S 1510

Nakashiro K 122

Nakayama T 1326

Narayan S 898

Nardini E 1964

Naylor B 1137

Neal DE 576, 1928

Negri E 341

Nelson P 350

Neoptolemos JP 1865

Neri P 1722

Neumaier C 1452

Neuman-Levin M 504

Newell DR 764

Neyns B 1592

Neyroud I 1251

$\mathrm{Ng}$ EH 1978

Ng EH-L 1978

Nguyen Bui B 1467

N'Guyen Bui B 1646

Nicholson RI 1958

Nicol A 741

Nicol DL 608

Nicoll G 1878

Nicolson M 137 (L)

Nieda M 741

Niederberger E 1585

Nieminen P 552

Nierenberg DW 683

Niitani H 939

Nijman RJM 1557

Niketeghad F 697

Ninane V 1444

Nishihara T 612

Nishino H 612

Nishioka K 412

Niyikiza C 649

Nobrega KM 1280

Noci I 333

Noda K 1064

Nonni AV 422

Noordzij MA 1557

Nordgren H 869

Nordin K 1265

Norman A 1258, 1624

Norman AR 692

Norris MD 1564

Nulman I 1611

Nyrén O 678, 1317

Oates J 692

Obara K 557, 1119

Oberlin O 1646

O’Byrne KJ 473, 863

Oda M 255

O’Donnell M 576

O'Donoghue DP 692

O'Dorisio MS 266

O’Dorisio TM 266

Ogawa S 1731

Ohira M 612

Ohira T 1706

Ohkusa T 692
Ohlsson L 129

Ohno Y 1634

Ohta Y 255

Okimoto N 9

Okuda H 93

Olaitan A 1824

Olschwang S 692

Olson W 1738

Olsson H 674

Omotehara F 122

Omura K 692

On On Chan A 1037

O'Nions J 1551

Ono Y 692

Onuki N 1510

Ormberg IW 1968

Ørntoft TF 568, 1211

Orosz C 285

Ortiz Reyes RM 46

Osakabe M 69

Oshikiri T 1922

Oshimura M 1510

Osin P 213

Osmond C 1680

Österreicher C 1462

Oswald J 1289

Ott RJ 1640

Ottonello L 463

Oude Luttikhuis MEM 531

Ouwehand M 1472

Ouyang N 1047

Ozaki K 247

Padhani AR 1624

Padovan E 107

Paesmans M 1444

Page A 1289

Pageaux J-F 1944

Paglierani M 333

Pahernik S 1655

Paik Y-K 1147

Papamichail M 1527

Papelard H 1347

Papini D 405

Park JH 1147

Parker C 1958

Parkes SE 350

Parkin D 242

Parmiani G 836

Parry L 1226

Pasini B 836

Pastuszko D 875

Patel A 1226

Pathak S 898

Patnick J 1289

Patterson LH 625

Patzelt T 1130

Paulsen T 74

Pauly M 692

Pavillard V 1077

Pawelec G 1527

Pearson A 705

Peaston AE 1564

Pedersen MW 1211

Pedrotti C 141

Péguet-Navarro J 1944
Pèlegrin A 1787

Pellizzaro C 795

Peng H 930

Pensotti V 845

Penz M 1462

Pera MF 608

Peretz T 36

Perey L 1713

Perez SA 1527

Perng R-P 1247

Perotti C 1794

Perret G 917

Perry R 705

Pesatori A 1304

Peters G 836

Peters R 1713

Petersen S 1372

Petrioli R 1722

Pezzella F 881

Philip T 1646

Phillips RM 1137

Phukan RK 661

Picton S 23

Pidgeon GP 273

Pierotti M 845

Pierotti MA 405, 836, 1535

Pilotti S 405, 1535

Pinder SE 225, 1958

Pinkerton CR 1746

Pinkerton R 831

Piris J 692

Pivot X 649

Plouet J 1944

Poli MA 141

Pollak M 428

Pont J 1462

Ponthan F 2004

Poon R 98

Poppe M 1801

Porrata LF 484

Portengen H 538

Postmus PE 1456

Potter JD 372

Poulsen HS 1211

Powell JE 350, 531

Powles R 325

Pozzessere D 1722

Prang J 1372

Preece PE 1157

Prehn JHM 1801

Prevost G 115

Price O 29

Price P 1085

Price SA 758

Pricolo VE 692

Primrose JN 1486

Pritchard-Jones K 831, 1746

Probst-Hensch NM 1695

Prochilo T 1452

Pruschy M 2010

Pugliese P 141

Puisieux A 902

Pujol JL 14

Pulimood AS 1928

Puolakkainen P 383 
Purdie CA 1102

Purdie D 1351

Purohit A 808

Pyle L 953

Qi K 1340

Qian L 930

Qiao Z 1332

Qiu WM 176

Quantin X 14

Queneau P-E 1251

Quirke P 692

Quoix E 808

Rabes HM 692

Raderer M 1462

Rades D 850

Radford J 29

Radice P 845

Radvanyi F 1515

Raitanen M-P 552

Rajpert-De Meyts E 220

Raju S 891

Rakowsky E 504

Ramani P 531

Rampaul RS 1958

Rampling D 493

Rampling R 1810 (BR)

Ranganathan S 735

Ranieri E 1738

Ranson M 909

Raouf A 1781

Rapallo A 692

Rath HC 1572

Rathi A 1510

Ravaud A 1467

Ray-Coquard I 816

Raymond E 649

Raymond L 1251

Reddy A 1551

Redfern D 350

Rees SA 531

Reimertz C 1801

Reinmuth N 584

Reitz M 1130

Remick DR 1600

Rettrup B 1871

Rewcastle NB 55

Rexrode KM 962

Rey JA 204

Reynolds JV 1499, 1781

Ricca A 1914

Riccardi A 141

Ricci P 379

Richard S 1077

Richardson DM 687

Riches AC 1157

Rieck G 546

Riecken E-O 1540, 1771

Riesterer O 2010

Rigg A 325

Rinaldi E 141

Rinnab L 1372

Rintala E 552

Ripoche V 649

Rissanen H 357
Ritchie AA 1753

Riva C 405, 1535

Rivera E 1396

Robert J 1077

Roberts MS 157

Robertson AJ 1157

Robertson JFR 1958

Robson CN 1928

Rochet N 1412

Rodolfo M 836

Roesch-Gateau O 902

Rogers L 1794

Rognum TO 692

Rohatiner A 29

Rolhion C 518

Rooney PH 1492

Rosell R 692

Rosenberg PS 1298

Rosing H 1472, 1627

Ross RK 1695

Rossi B 1412

Rosso R 1452

Roth T 1585

Roufogalis BD 1998

Rovini D 836

Rozendaal L 398

Rozycka M 1551

Rubin G 1289

Rudas M 1764

Rudin C 325

Rumio C 1964

Rümmele P 1572

Runsink AP 398

Russell S 1432

Russo A 692

Ryan BM 1499

Rylander E 1153

S-1 Cooperative Study Group 939

Saarialho-Kere U 383

Sabatino M 1722

Sabokbar A 78

Sadanaga N 713

Sadetzki S 1368

Saga S 563

Sagawa M 1326

Saint Frison M 1831

Saito K 1119

Saito Y 1032

Sakaguchi H 313

Sakai S 1634

Sakata K 69

Sakurai T 1006

Salamon E 1

Sällström J 1153

Salmela MT 383

Saltnes T 1478

Sampson JR 1226

Sandblom G 497

Sann H 924

Santos C 966

Sappino AP 1251

Sapunar F 953

Sarasa JL 204

Sasaki T 255
Sasano H 1731

Saso R 325

Satchi R 1070

Sato C 228

Sato K 199

Sato M 122

Sato S 1032

Saunders MI 1113

Saurin JC 721

Sauter ER 1952

Sawada T 612

Saxby MF 1504

Scheidtmann KH 1801

Schellens JHM 1472

Scherübl H 1771

Schirmacher P 697

Schmidinger M 1850

Schmidt C 741

Schmitt C 1646

Schmitt D 1944

Schneider M 649

Schneider PM 1168

Schölmerich J 1572

Schot ME 1472

Schott H 1130

Schradin T 1372

Schramel FMNH 1456

Schulte K 1585

Schulz WA 1887

Schumm-Draeger PM 1546

Schuppan D 1540

Schuyer M 1359

Sciot R 98

Scoazec JY 721

Scorilas A 190

Scott D 293

Scott N 787

Scottish Cancer Therapy

Network 637

Scotton C 891

Screnci D 1219

Sculier JP 1444

Sebban C 816

Segawa Y 9, 939

Seifeldin IA 1037

Seifert H-H 1887

Seiler M 107

Seki N 93

Sekirnik A 1193

Selby P 1842

Semenciw R 1335

Senagore AJ 692

Serve H 1168

Sette A 1738

Seyfried TN 285

Sfondrini L 1964

Shah TK 1137

Shaheen RM 584

Shamma A 412

Shankar A 1759

Shaper AG 1311

Sharkey I 23

Sharp L 1667

Sharrard RM 590

Shaw D 129

Shelling AN 687
Shen N 930

Shennan MG 527

Shi R 991

Shibayama T 9

Shibuya K 431

Shimada Y 1006

Shimamura H 431

Shimokata K 1634

Shinohara T 1922

Shiozaki H 412

Shipley J 213, 831

Shou Y 1706

Shtoyerman-Chen R 1368

Shu X-O 372

Sidiropoulos M 393

Sidney J 1738

Siebert GA 157

Siebrands C 850

Siegfried JM 235

Sigsjö P 497

Simon J 1372

Simonato L 341

Singhal S 325

Sinha D 337

Sirohi B 325

Sitas F 1322

Sivridis E 881

Skawran B 850

Slingluff CL Jr 1738

Sliutz G 791

Smalley K 953

Smedshammer L 1900

Smeets SJ 630

Smeland EB 1900

Smit EF 1456

Smith A 1842

Smith DR 692, 1812

Smith HJ 297

Smith J 1824

Smithers BM 157

Smyth JF 944, 1753

Snary D 692

Snijders PJF 398

Snow GB 630

Sobin LH 780 (L)

Soliman AS 1037

Soliman EH 1557

Song E 1047

Sotelo J 1396

Sotiriadou R 1527

Sotiropoulou PA 1527

Soufir N 1883

Souglakos J 798

Sozzi G 405, 1535

Spagnoli GC 107

Sparreboom A 1099, 1124

Spencer SK 683

Spiro SG 1609 (BR)

Spreadborough A 293

Staff S 1201

Stanczyk FZ 1695

Staquet M-J 1944

Stark A 1865

Stark M 590

Starzec A 917

Stassar MJJG 1372 
Stebbing J 953

Steel CM 1157

Steel J 1265

Steen HB 1900

Steger GG 1850

Stegmaier C 972

Steininger R 1850

Steinmann D 850

Stern PL 129

Stevenson DAJ 1492

Stewart F 937

Stewart M 1937

Stewart ME 944

Stockton D 41

Stokke T 1900

Stora de Novion H 1412

Storkus WJ 1738

Stoter G 1099, 1359

Straetmans N 1387

Strand A 1153

Strange RC 1504

Stremmer A 1193

Streuli CH 1522

Strickler HD 1295

Strong V 1842

Struewing JP 527

Stumbo M 1535

Sturdy L 1289

Stürmer T 972

Stuttgen MA 608

Stylianou K 798

Su F 1047

Suarez H 1831

Sudaryo MK 1557

Sugimachi K 713

Sugita M 64

Sulkes A 504, 523, 1368

Sullivan A 1551

Summersgill B 213

Sun X-L 930

Sun XF 692

Sunamura M 431

Sutton G 1289

Suzuki T 1731

Suzuoki M 1922

Sweep CGJ 85

Sweneey E 1781

Swerdlow AJ 1332

Taal BG 1437

Tabata M 9

Tabone E 902

Taddei GL 333

Tadokoro K 741

Tagnon A 1

Takahashi K 557, 1119

Takano T 102

Takata I 9

Takeda K 431

Takigawa N 9

Talks K 881

Tamaya T 313

Tamborini E 405, 1535

Tamir A 1368

Tammela TLJ 552

Tamura G 69, 199
Tamura M 255

Tamura S 412

Tan MG-K 1978

Tanaka F 713

Tanaka M 692

Tanaka N 64

Tanaka Y 255

Tang WC 1585

Tanière $\mathrm{P} 721$

Tanimoto M 9

Tanner MM 1201

Tattersall MHN 1273

Taylor I 1759

Tchekmedyian S 152

Tchirkov A 518

Teas J 372

Tejpar S 98

Ten Bokkel Huinink WW 1472

Teraoka H 612

Terrier P 1646

Terry P 346

Teugels E 1592

Thatcher N 137 (L)

Thebo JS 692

Theodor L 1368

Thiery JP 1515

Thiriaux J 1444

Thomas V 171

Thompson AM 1102, 1157, 1878

Thompson JF 157

Thompson MG 297

Thomson CS 637

Thomson JZ 225

Thykjaer T 568

Thykjær T 1211

Tibaldi C 1452

Tidy JA 1551

Tiffin N 1746

Tinelli C 141

Tisdale MJ 297, 758

Tollenaar RAEM 1347

Tomasic G 795

Tomek S 1764

Tomita Y 557, 1119

Tomkins SE 453

Torrisi R 1838

Tortolina G 463

Traub T 1462

Travis WD 1510

Treleaven J 325

Trinca S 379

Trisciuoglio D 1914

Trotti G 141

Troungos C 692

Tseng WW 584

Tsuchida S 1032

Tsuchiya F 1731

Tsuchiya T 69, 199

Tsukada H 1326

Tsunezuka Y 255

Tucker MA 527, 1304

Tuomilehto J 1680

Turley H 261, 881

Turner R 1340

Twelves CJ 637

Tytgat J 1
Uchida D 122

Ueda M 64

Ueki M 64

Uemura H 563

Ueoka $\mathrm{H} 9$

Ugnat A-M 1335

UK Cancer Cytogenetics

Group 831

UK Childhood Cance Study Investigators 1685

UK Children's Cancer Study Group 831

Ukena D 1130

Uleryk E 1611

Umemoto M 1032

Urosevic N 692

Usadel KH 1546

Usel M 1251

Usuba O 199

Vaccarella S 46

Valagussa P 490

Valavanis C 692

Vamvakas L 798

van Belle $S 1$

Van Camp B 1387

Van Cutsem E 98

Van Cutsem O 1444

van de Ouweland AMW 538

van de Vijver MJ 1347

van den Brandt PA 977

Van den Heuvel MM 1557

van der Burg MEL 1124, 1359

Van der Kwast TH 1557

van der Linden HC 398

van der Zee AGJ 1627

van Ee CC 687

Van Hul W 176

Van Krinks CH 453

van Lier JE 1787

Van Riet I 1387

van Staveren IL 538

van Steenbrugge GJ 1557

Van Tellingen O 1472

van Tienoven $\mathrm{ThH} 85$

Van Tinteren $\mathrm{H} 1437$

Vande Broek I 1387

Vanderkerken K 1387

Vandervellen R 1

Vanier-Viornery A 902

Varani J 1600

Varenhorst E 497

Varley JM 293

Vassy R 917

Vatten LJ 959

Velarde C 966

Velikova G 1842

Venutolo E 1883

Verhoog L 538

Vermeij J 1592

Veronesi G 1838

Verrelle P 518

Verweij J 1099, 1124

Villa E 1607 (L)

Villette JM 590

Vilmer C 1883
Vindevoghel A 1

Virgolini I 1462

Virmani A 1510

Voelter W 1527

Vogt U 1168

von der Linden GHM 1099

von Jürgenson S 1130

von Schlippe M 823

Voorhorst FJ 398

Voorzanger-Rousselot N 902

Vousden KH 1813

Vuong V 2010

Wadler S 692

Wagner J 1295

Wakai K 1634

Wakai S 1326

Walboomers J 966

Walker M 1311

Walker RA 618, 863

Wallace H 23

Waller DA 863

Wallgren A 362

Walsh T 692

Wandert T 1130

Wang G-S 1247

Wang H-Y 1162

Wang J 176

Wang LG 1403

Wang M 1047

Wang ST 692

Wang Y-S 1185

Wang Z-X 1162

Wannamethee SG 1311

Ward R 692

Wardman G 1289

Warin AP 803

Wärnberg F 869

Wascher RA 1340

Watanabe G 255

Watanabe M 228

Watanabe N 752

Watanabe T 1037

Watanabe Y 9, 1064

Waters JS 1258

Watson JC 266

Watson M 166

Watson PF 875

Watters AD 1894

Webb A 1258

Webley SD 446

Weeks J 1824

Weetman AP 875

Weiner D 1206

Weinreb M 350

Weisinger G 1771

Weizman A 1771

Welsh SJ 446

Wen B 2017

Wencyk PM 1958

Wenzel C 1850

Werle B 1193

Wessel N 1418 (L)

West AF 576

West J 64

White J 803 
Whitehead SM 803

Wickramasinghe C 705

Wilander E 1153

Wilkinson K 692

Will BP 1280

Willemse PHB 1627

Willhardt I 924

Williams ARW 1023

Wilms E 1124

Wilson A 29

Wilson ARM 225

Wilson J 891

Wilson R 1289

Wiltschke C 1764

Winder R 1289

Wingren S 859

Winn R 64

Winqvist R 209

Winship IM 687

Wloch J 875

Wolf CR 1175

Wolfson MC 1280

Wolk A 346, 984

Woltering EA 266

Wolters M 791
Wonderling D 166

Wong J 1206

Wong NACS 1937

Wood J 2010

Woodman CBJ 787

Workman P 1085

Wright P 1842

Wroughton MA 803

Wu D-C 658

Wu H 176

Wu L 261

Wu M-C 1162

Wu M-T 658

Wuyts W 176

Xiao CY 176

Yagihashi A 752

Yajima T 752

Yamaguchi H 713

Yamamoto H 412

Yamasaki M 247

Yamauchi J 431

Yang B-C 1185

Yang K-Y 1247
Yang P-W 658

Yano M 412

Yashiro M 612

Yasuda T 412

Yates AJ 285

Ye W 678, 1317

Yokoyama A 1326

Yokoyama Y 1032

Yoshida H 102, 122

Yoshida K 563, 1706

Yoshikawa K 563

Yoshimori K 939

Yoshino K 247

Yoshinouchi M 93

Young J 692

Young LS 350

Yu C 98

Yu H 991

Yu MC 1695

Yuan J-M 1695

Yuen ST 692

Zafrani ES 1515

Zanghellini E 1412

Zeegers MPA 977
Zeilstra LJW 1055

Zeinoun Z 1592

Zeitz M 1771

Zellweger M 1251

Zhang G 176

Zhang H 692

Zhang J-C 428

Zhang SZ 176

Zhao F 1706

Zheng W 372

Zhou Z 747

Zhu D 1037

Ziegler H 972

Zielinski CC 1764

Zielinski GD 398

Zietz C 692

Ziyaie D 1102

Zoetmulder FAN 1437

Zöller M 1372

Zupi G 1914

Zweit J 1640

Zwelling LA 747 